\title{
Mesocrystals of Vanadium Pentoxide: A Comparative Evaluation of Three Different Pathways of Mesocrystal Synthesis from Tactosol Precursors
}

\author{
Christine Lausser, Helmut Cölfen, ${ }^{t, *}$ and Markus Antonietti \\ Max-Planck-Institute of Colloids and Interfaces, Colloid Chemistry, Research Campus Golm, Am Mühlenberg, D-14424 Potsdam, Germany. ${ }^{\dagger}$ Present address: University of \\ Konstanz, Physical Chemistry, Universitätsstr. 10, D-78457 Konstanz, Germany.
}

$M$ esocrystals have gained major interest in various fields of research in the past few years. ${ }^{1}$ The term "mesocrystal" is an abbreviation for a mesoscopically structured crystal. ${ }^{2} \mathrm{~A}$ mesocrystal is constituted from crystalline nanoparticles which are often arranged in a perfect three-dimensional way. ${ }^{2-4}$ In this respect, the scattering behavior in X-ray and electron diffraction of such a structure is similar to that of a single crystal. Therefore, it is sometimes difficult to distinguish between the presence of a single crystal and a mesocrystal. Nevertheless, in the past few years, the number of detected and established cases of mesocrystal formation rose, also due to the improved possibilities of modern structure analysis. ${ }^{2-7}$

The mesocrystal concept applies especially in the field of biomineralization. Natural organisms have routes to control the shape and size of inorganic nanocrystals for optimized storage and transport and can later arrange them into ordered superstructures with unique properties. ${ }^{8-15}$ Hence, many scientists have tried to study and mimic the biomineralization processes with the goal to synthesize such crystalline superstructures. ${ }^{16-21}$ However, the formation of mesocrystals is not limited to biomineralization, and meanwhile, a large number of purely synthetic mesocrystals were synthesized successfully. ${ }^{1,2,22-24}$ In particular, mesocrystals of functional inorganic materials are of high interest, as they exhibit improved material properties. These are, for instance, the combination of high crystallinity with high inner surface area while providing a larger scaffolded superstructure at the same time. Many mesocrystals offer the www.acsnano.org
ABSTRACT Vanadium pentoxide mesocrystals were synthesized from a mineral liquid crystalline precursor phase, a so-called tactosol. For comparative evaluation of solid formation from that phase, the distance between the vanadium pentoxide particles was lowered by three different modes: (a) by adding a $0.1 \mathrm{M} \mathrm{NaCl}$ solution, the electric double layer was compressed and controlled particle aggregation was induced; (b) application of external pressure by ultracentrifugation resulted in particle compression and final crystallization; (c) an acrylic acid/ sulfonic acid copolymer was added to introduce polymer-mediated particle alignment and densification. In all three cases, the preorientation of the particles within the liquid crystal remained, and different mesocrystals were formed. This was demonstrated by comparative analysis of the resulting structures by scanning electron microscopy (SEM), transmission electron microscopy (TEM), and polarization microscopy.

KEYWORDS: mesocrystal $\cdot$ vanadium pentoxide $\cdot$ liquid crystal $\cdot$ tactoid $\cdot$ fibers

high surface area of the constituting nanoparticles but can be separated like a macroscopic object (i.e., by filtration). This combination is, for instance, advantageous for catalytically active materials. In this study, we investigate mesocrystals of vanadium pentoxide $\left(\mathrm{V}_{2} \mathrm{O}_{5}\right)$, which is widely used as catalys $\mathrm{t}^{25-29}$ and therefore relevant for industry.

In the present work, we use a vanadium pentoxide tactosol as a model case and starting point for the reconstitution toward mesocrystals. The tactoid sol phase of the $\mathrm{V}_{2} \mathrm{O}_{5}-\mathrm{H}_{2} \mathrm{O}$ system was discovered in the 1920 s by Zocher ${ }^{30-33}$ and is chemically classified as a lyotropic, inorganic, nematic liquid crystal. ${ }^{34}$ The tactoids themselves are built up by rod-shaped small particles which are likewise mutually oriented in a nematic and anisotropic manner. ${ }^{35}$

Here, we use preorientation of the nanoparticle building units in the tactoid sol to realize a simplified starting situation for the formation of mesocrystals. By minimizing their repulsive forces, the tactoids as well as the primary $\mathrm{V}_{2} \mathrm{O}_{5}$ particles, which form the
*Address correspondence to helmut.coelfen@uni-konstanz.de.

Received for review July 21, 2010 and accepted December 15, 2010.

Published online January 4, 2011. 10.1021/nn1017186

() 2011 American Chemical Society 


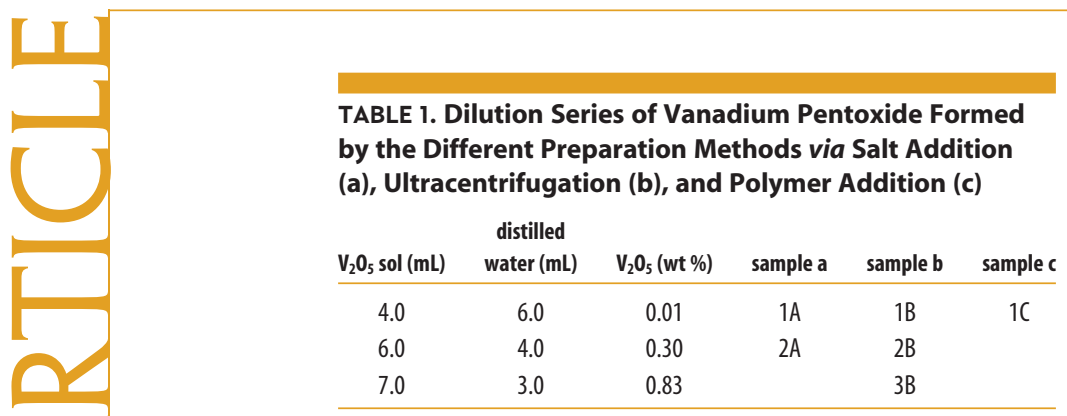

tactoids, can be merged. ${ }^{36}$ The distance between the particles to induce mesocrystallization was lowered by three different modes: (a) adding an electrolyte in form of $\mathrm{NaCl}$ solutions, (b) application of external pressure via ultracentrifugation, and (c) adding an interacting polymer. As a result, mesocrystals of this functional material could be easily synthesized in all three cases, with slight variation of their inner structure.

\section{RESULTS AND DISCUSSION}

For the following experiments, the subsequent dilution series of vanadium pentoxide tactosols are presented in Table 1.

Addition of Sodium Chloride Solution. Already screening of the repulsive interactions by salt addition gave homogeneous, pillow-like superstructures with rounded edges and porous surfaces. Scanning electron microscopy (SEM), transmission electron microscopy (TEM), electron diffraction (ED), and polarization microscopy (PLM) examined the microstructure of the final products. Observations of the product with SEM (Figure 1a) show rectangular structures with rough surfaces and curvature. These are the typical hints that the structures are mesocrystals.
TEM of thinner species (thickness about $80 \mathrm{~nm}$ ) shows that the former tactoid rods have rearranged to form thin platelets, which again pack to larger stabs (Figure 1b). The platelets are built up by the primary rod-like precursor nanoparticles of the tactoids with small dimension of ca. $10-20 \mathrm{~nm}$ as estimated from the TEM micrograph in Figure 1b. This causes an inner structure that can be observed in the TEM measurement. Electron diffraction on such a thin specimen (Figure 1c) indicates, with potentially some twinning, the presence of a crystalline superstructure. This is confirmed by polarization microscopy (Figure 1d) where, besides a pronounced inner pore structure of the "pillows", isorefractive orientation was observed even on the longer distances of up to a few micrometers accessible to light microscopy.

The distances between the primary $\mathrm{V}_{2} \mathrm{O}_{5}$ nanorods in the tactoids resulting from electrostatic stabilization were reduced by adding the $\mathrm{NaCl}$ solution. The electric double layer shrinks; a final $56.5 \mathrm{mM} \mathrm{NaCl}$ solution corresponds to a Debye length of $1.3 \mathrm{~nm}$. Experimentally, this is the distance where the attractive van der Waals forces take over, leading to the controlled coagulation and alignment of the primary nanoparticles. Semiquantitative DLVO calculations of the interaction potential between two spherical $\mathrm{V}_{2} \mathrm{O}_{5}$ nanoparticles underline this statement. The $\xi$-potential of the $\mathrm{V}_{2} \mathrm{O}_{5}$ nanoparticles in water was determined to be $-70 \mathrm{mV}(\mathrm{pH} 4.2)$. These nanoparticles are stable since the primary maximum in the particle interaction potential is high with 25 $\mathrm{kT} / \mathrm{nm}$. However, in case of the tactsol, the $\xi$-potential is decreased to $-56 \mathrm{mV}$. In this case, the interaction potential between the nanoparticles is $13 \mathrm{kT} / \mathrm{nm}$, as can be seen in Figure 2. Under these conditions, approach-
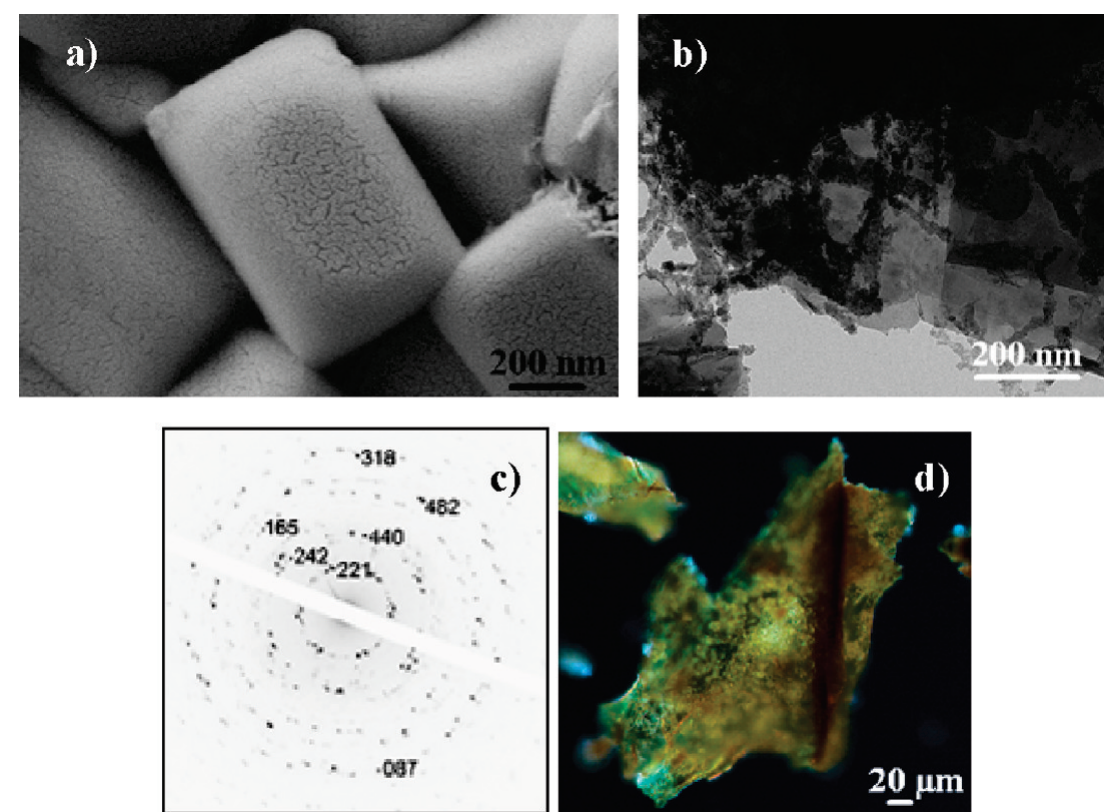

Figure 1. (a) SEM image showing rectangular structures of sample 1A. (b) TEM image of sample 1A. (c) Electron diffraction shows a spot image as it is obtained for ordered structures. Using a polarization microscope with crossed polarizers, the inner long-range order of the particles could be depicted as areas with similar color (d). 


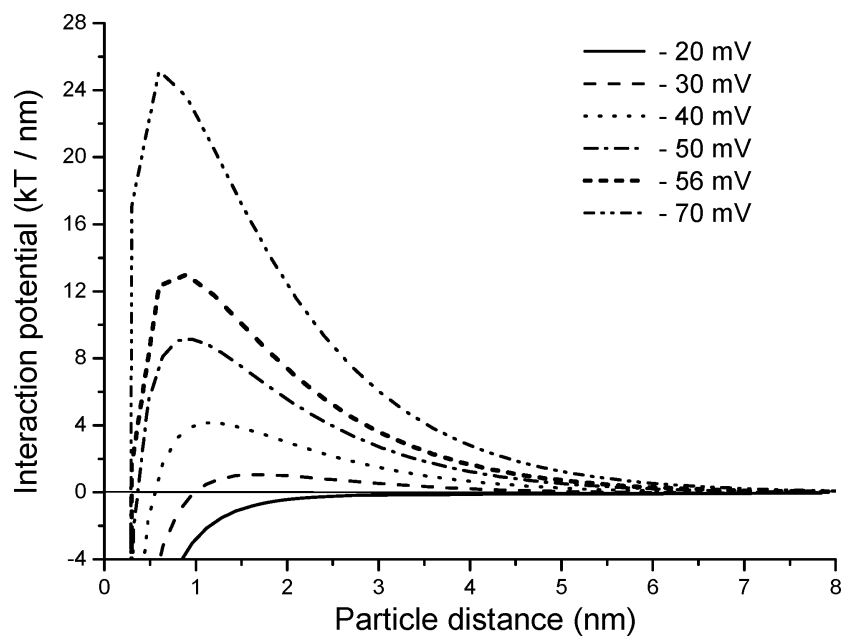

Figure 2. Interaction potential between two spherical $\mathrm{V}_{2} \mathrm{O}_{5}$ nanoparticles $(d=13 \mathrm{~nm})$ with different $\xi$-potentials depending on their distance according to the DLVO theory calculated with the program Hamaker. Parameters: $\mathrm{V}_{2} \mathrm{O}_{5}$ density $=3.357 \mathrm{~g} / \mathrm{mL}$, Hamaker constant for $\mathrm{Na}^{+}$intercalated $\mathrm{V}_{2} \mathrm{O}_{5}=7.9 \times 10^{-20}($ ref 37), $\xi$-potential plane $=0.5$ $\mathrm{nm}$, dielectric constant solvent $=78.54,\left[\mathrm{Na}^{+}\right]$and $\left[\mathrm{Cl}^{-}\right]=56.7 \mathrm{mM}$.

ing nanoparticles are stable enough that they can optimize their mutual orientation before mesocrystal formation. It must be noted that the calculations are not quantitative since $\mathrm{V}_{2} \mathrm{O}_{5}$ nanoparticles are platelets for which quantitative DLVO calculations are not yet possible, but they support our observations.

Adding $\mathrm{NaCl}$ solution to samples with higher sol concentration (sample 2A) leads to crystalline superstructures, as well. However, the shape of the crystals is not as defined as in sample 1A: in SEM, the superstructures are much more rounded, still droplet-like (Figure 3a). TEM on a specimen with a thickness of about $80 \mathrm{~nm}$ indicated more open nets of primary nanoparticles with larger interstitial pores (Figure $3 b)$. The tactoids did not rearrange to thin discs stacking together as in sample 1A. Fusion is obviously so fast that they rather fuse to a larger droplet-like particle. Anyway, the individual precursor nanoparticles of the tactoids could be still identified with sizes estimated to be $10-20 \mathrm{~nm}$. Local ED, however, still reveals a single-crystal-like alignment of nanoparticles (Figure 3c). On the larger micrometer scales covered by polarization microscopy, a common joint alignment is missing: the big droplet particles seem to be composed of a few domains, each of which displays single crystalline order, however with inner structure and porosity.

Apparently, the higher concentration of the tactoids influences the structure formation process. On the basis of the higher concentration of nanoparticles, the coagulation is faster and only lower mutual organization of the particles can be accomplished. Presumably, each individual tactoid forms single mesocrystals, which subsequently aggregate to the larger multidomain structures.

Mesocrystals via Ultracentrifugation of a Tactosol. The distance between the $\mathrm{V}_{2} \mathrm{O}_{5}$ particles can be also reduced by application of an external centrifugal force, here conveniently applied in an ultracentrifuge. Samples with different sol concentrations were exerted to the full centrifugal field of $367598 \mathrm{~g}$. After ultracentrifugation, all samples consist of macroscopic, crystalline fibers, which form a densely packed superstructure layered one upon the other. It is to be underlined that the primary tactoid is the same; that is, the morphology differ-
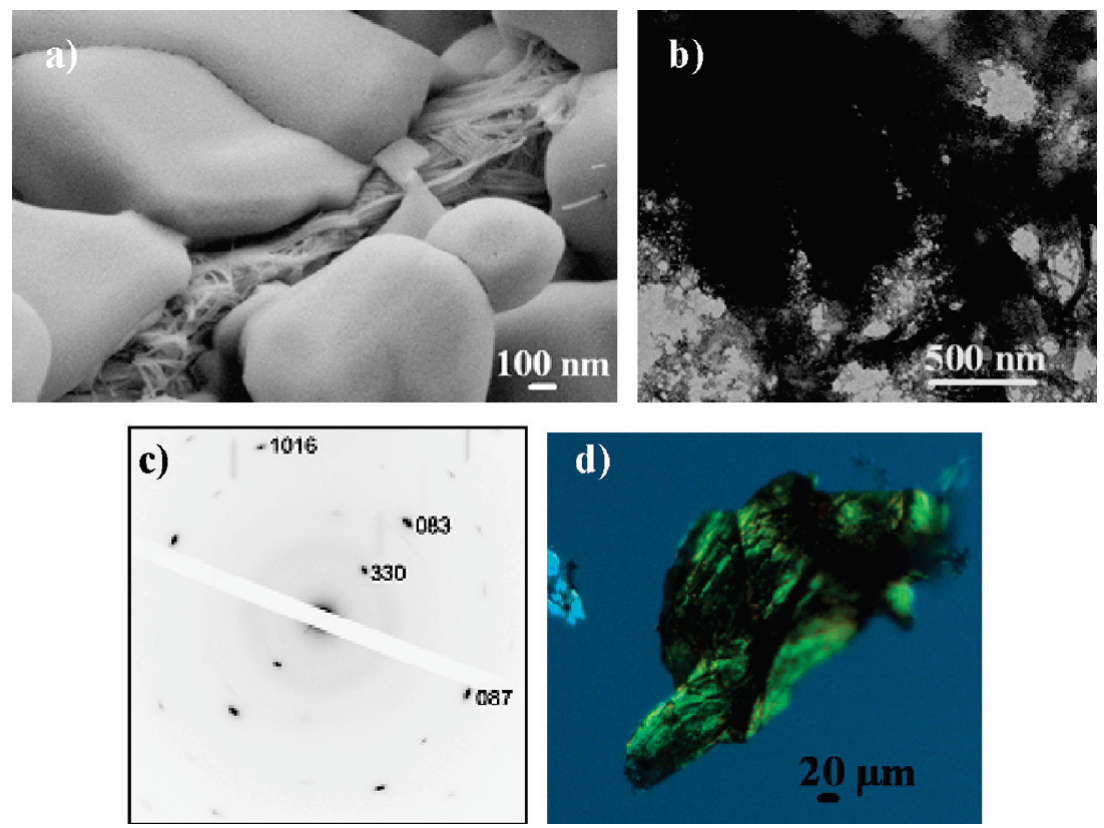

Figure 3. (a) SEM images of sample 2A; TEM and SAED measurements of sample 2A demonstrate the short-range order of the particles within the crystals $(b, c)$. (d) Polarization microscopy with crossed polarizers indicates long-range order of the particles in the range of several micrometers inside the samples. 


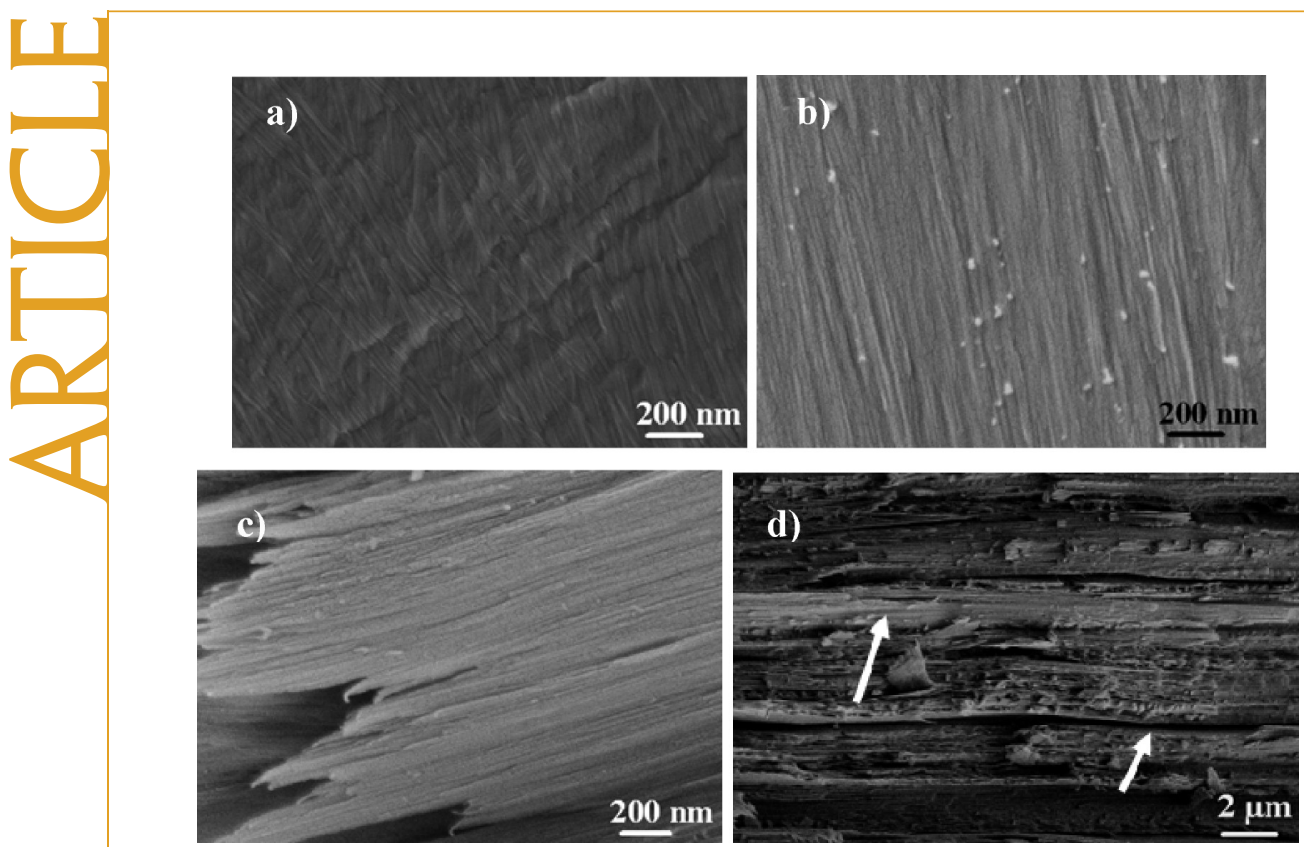

Figure 4. SEM images of samples obtained after ultracentrifugation. (a) In sample 1B, the secondary fibers are still slightly misaligned. (b) For the other samples with higher $\mathrm{V}_{2} \mathrm{O}_{5}$ concentration, it is observed that the fibers are layered one upon the other in mutual alignment (exemplarily, sample 2B is shown). (c) Final mesocrystals are made by layers of fibers (image of sample 2B). (d) Layers stack parallel to each other, as it can be visualized for sample 3B (examples marked by arrows).

ences are indeed just due to the different control of mesocrystallization.

Already for the sample with the lowest concentration, sample 1B, we found very long, extended crystalline fibers, which however are mutually still slightly misoriented (Figure 4a). This is different for the higher concentrations where a close to perfect alignment of the fibers was observed.

Obviously, concentration has a positive influence here. While the concentration of the tactoids of sample $1 \mathrm{~B}$ is somewhat too low for perfect alignment (and presumably keeps the liquid crystalline order of the tactoids), the other samples can align close to perfection. In those samples, the fibers build up layers stacked parallel to each other. This layering arises from the posi-
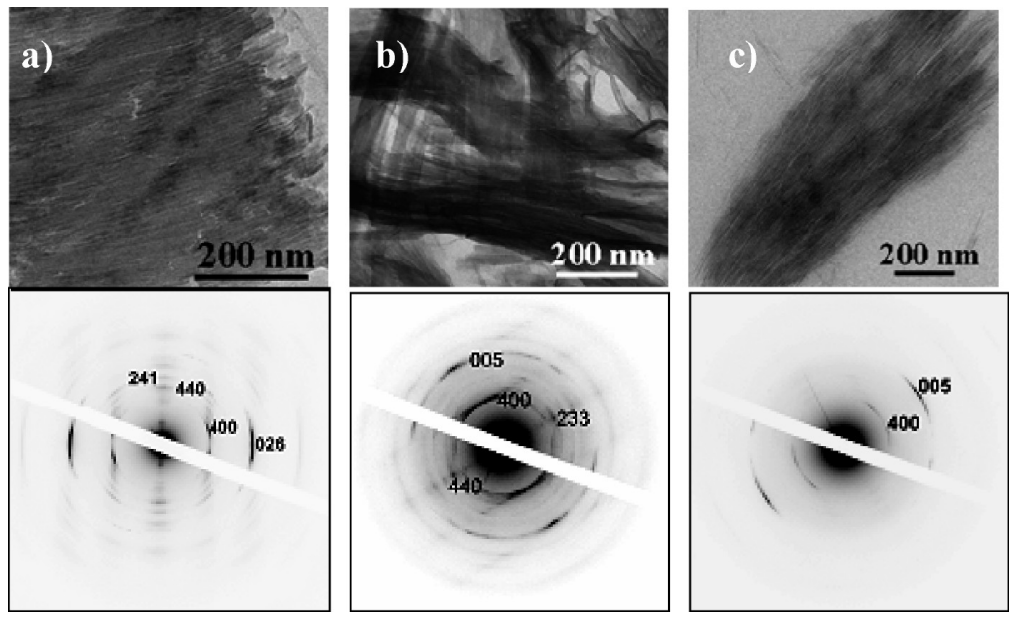

Figure 5. TEM images of the samples 3B (a), 2B (b), and 1B (c) obtained after ultracentrifugation: the electron diffraction shows a short-range order of the fibers. tion of the $\mathrm{V}_{2} \mathrm{O}_{5}$ nanoparticles inside the tactosol. Such filaments were already described in previous work on vanadium pentoxide tactosols ${ }^{34,38}$ and were found to be superstructures of nanoparticles, themselves. We can support this view from high-resolution SEM pictures showing fibers in parallel alignment with some of the building units still visible on the fibers in Figure $4 b$. The processes induced by ultracentrifugation are presumably similar to those, which occur during the process of drying or aging of the tactosol since both lead to concentration increase of the nanoparticles supporting superstructure formation. Small $\mathrm{V}_{2} \mathrm{O}_{5}$ particles show oriented aggregation and recrystallize to elongated rods at the same time. Friction during sedimentation leads to further mutual alignment of the rods. It was speculated that vanadic acid, generated by hydrolysis, is adsorbed more at the sides of the $\mathrm{V}_{2} \mathrm{O}_{5}$ particles than at the end, ${ }^{39}$ which would explain the formation of fibers by oriented attachment with subsequent particle fusion. These rods then again arrange to densely packed, crystalline patterns.

The arrangement of the nanoparticles within the tactoids as well as the orientation of the formed fibers can also be characterized by TEM. In all three samples, oriented primary nanofibers with different degree of mutual order are clearly visualized. The spots of electron diffraction are here deformed to arcs, indicating a slight misalignment in the vectorial alignment of the superstructure. The fibers are bent in some areas (as seen in Figure 5b). However, we cannot exclude that the minor misorientation of the fibers is an artifact due to mechanical stresses throughout the preparation of the TEM samples. The findings at least prove that the primary fibers can be easily separated from each other.

Polarization microscopy demonstrates the single-crystal-like, long-range order of the fibers (data not shown here). BET measurements of samples $1 \mathrm{~A}, 2 \mathrm{~A}, 2 \mathrm{~B}$, and $3 \mathrm{~B}$ show a moderate increase of the surface area as compared to an industrial $\mathrm{V}_{2} \mathrm{O}_{5}$ powder provided by Aldrich (Table 2). This is a second proof that the higher tectonic units of the mesocrystals do not fuse or recrystallize throughout this process, and a porous material can be obtained. This is potentially useful for the catalytic applications of $\mathrm{V}_{2} \mathrm{O}_{5}$. 
TABLE 2. Results of the BET Measurements on Some Mesocrystals (Samples Were Gassed out at $400^{\circ} \mathrm{C}$ for $20 \mathrm{~h}$ )

\begin{tabular}{cccccc} 
sample & $\mathbf{V}_{\mathbf{2}} \mathbf{0}_{\mathbf{5}}$ powder & $\mathbf{1 A}$ & $\mathbf{2 A}$ & $\mathbf{2 B}$ & $\mathbf{3 B}$ \\
\hline surface area $\left(\mathrm{m}^{2} / \mathrm{g}\right)$ & 7.8 & 11.4 & 14.4 & 12.8 & 13.8
\end{tabular}

Influence of the Polymer ACUSOL 588G. It is the basic idea of polymer-controlled mesocrystal formation that the mutual interaction potentials of the primary nanoparticles can be also modified by addition of a surface-active polymer. Here, we used the commercial mineral stabilizer ACUSOL 588G. Details of this polymer are found in the Materials and Methods.

Indeed, it was observed that addition of polymer resulted in slow formation of larger crystalline superstructures. SEM analysis of the sample shows curved objects built of extended stacks of plates (Figure $6 a, b$ ). TEM of specimen with a thickness below $100 \mathrm{~nm}$ obviously depicts the stacking of several platelets (Figure $6 d)$. ED of these samples confirms the presence of superstructures with high mutual order of the building blocks (Figure 6c). In addition, polarization microscopy observations (Figure 6e) demonstrate long-range order in the product by showing regions of the same color in the micrometer range. The nanoparticle superstruc-

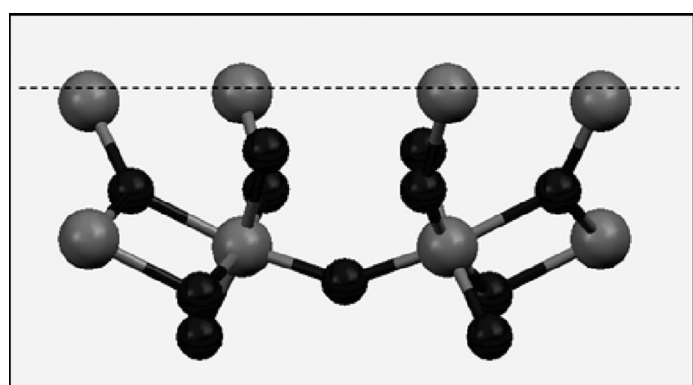

Figure 7. At the $\{020\}$ face (dashed line), either vanadium (dark) or oxygen (gray, as shown) can be exposed at the surface.

tures contain a high amount of polymer of 11.9 wt \% as determined by thermogravimetry.

At the $\mathrm{pH}$ value of the tactosol of about 4.2, the major part of ACUSOL 588G is protonated (except the 14.7 mol \% sulfonate groups). This supports the binding via hydrogen bridges, while the sulfonates keep the polymer in solution. In the direction of the $\{020\}$ face, vanadium pentoxide exposes the $\mathrm{V}=\mathrm{O}$ group with two electron pairs at the oxygen atom (Figure 7), which are strong hydrogen bridge acceptors, leading speculatively to preferential polymer binding to $\{020\}$ since $\{020\}$ is the known exposed face for $\mathrm{V}_{2} \mathrm{O}_{5}$ layers. ${ }^{34}$ The
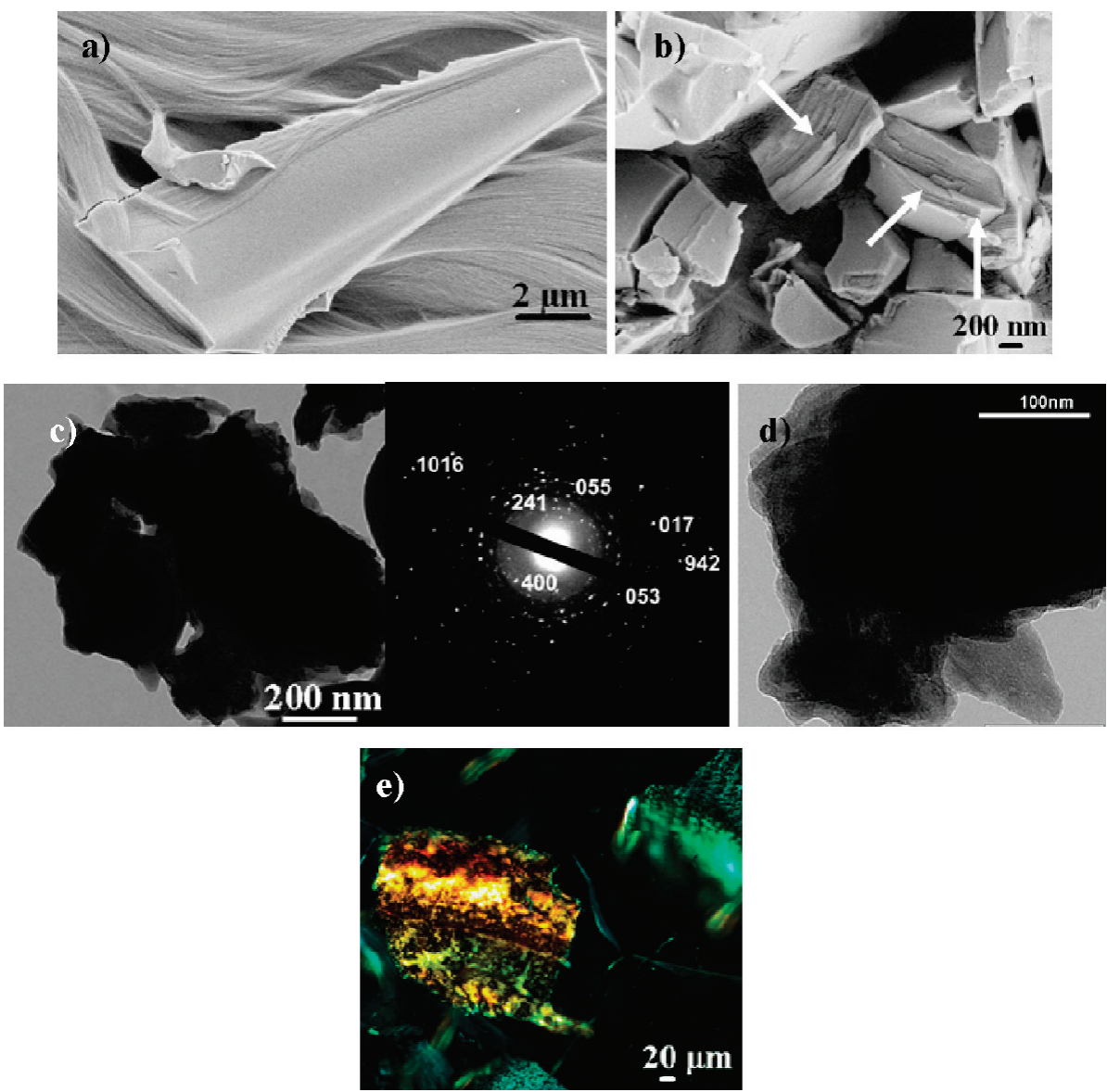

Figure 6. (a,b) SEM images of a $\mathrm{V}_{2} \mathrm{O}_{5}$ tactosol after addition of ACUSOL 588G. Small plates form, which further assembled into layers. (c,d) TEM image of the small plates. They show spot-like electron diffraction, indicating mutual organization. (e) Polarization microscopy image: there is a long-range order over several micrometersdetected throughout the hierarchical assembly of particles. 


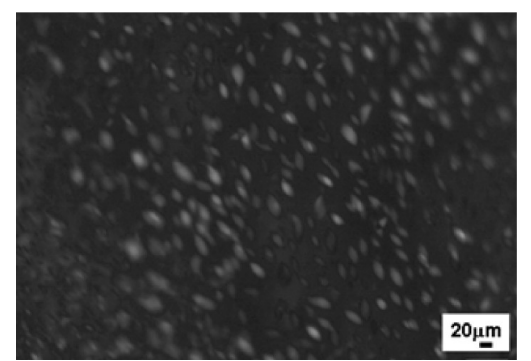

Figure 8. Polarization microscope image of a $\mathrm{V}_{2} \mathrm{O}_{5}$ tactosol after about 2 weeks.

growth in the $\{020\}$ direction is thereby blocked, and this is how it can be explained why this surface is texturally expressed in the presence of a carboxylate.

Measurements by analytical ultracentrifugation show primary $\mathrm{V}_{2} \mathrm{O}_{5}$ particles within the tactosol on the scale of $13 \mathrm{~nm}$ hydrodynamic diameter in agreement with the estimated size of $10-20 \mathrm{~nm}$ from TEM (Figures $1 \mathrm{~b}$ and $3 \mathrm{~b}$ ). These nanoparticles first form nanofibers by interaction, which are nematically arranged in form of the spindle-shaped tactoids with an average size in the order of $20 \mu \mathrm{m}$ (see Figure 8). The polymer has a contour length of about $40 \mathrm{~nm}$ and can therefore bridge some of the preorganized $\mathrm{V}_{2} \mathrm{O}_{5}$ nanorods to form the $\mathrm{V}_{2} \mathrm{O}_{5}$ layers. By adsorption of the polymer, and the ability to form hydrogen bonds to adjacent nanocrystal layers, extended mesocrystals are formed by stacking, with polymer sandwiched between the nanoparticles exposing $\{020\}$ faces (Scheme 1).

The electron diffraction of the plates shows a spotlike image, which is typical for single crystalline behavior. However, the habitus of some particles looks like there are varying orientations inside the mesocrystalline texture.

The SEM measurement depicts obviously the stacking of several shafts within the final superstructure (Figure $6 \mathrm{~b}$, marked by arrows). The plates show birefringence under crossed polarizers. However, the color of the reflected light is not uniform. It seems that the glimmering plates are composed of a small number of areas with single crystalline order, but certainly do contain inner structure, like a pore system.

\section{CONCLUSION}

In summary, it was demonstrated that the preorientation of inorganic colloidal nanoparticles into a mineral liquid crystal is a favorable starting point to enable and study formation of a structural variety of mesocrystals. A primary, stable vanadium pentoxide tactosol was densified in three different simple ways, and three different mesocrystal structures were found. First, salt was added to decrease the Debye length of the electric double layer stabilizing the $\mathrm{V}_{2} \mathrm{O}_{5}$ sol. An ordered agglomeration took place, which led to pillow-like, threedimensional, porous superstructures. The alignment of primary particles on a local scale was, however, found to be far from perfect, and depending on concentration, more or less extending porosity was included in the otherwise well-shaped mesocrystals. We conclude that salt addition is presumably the least controlled way to promote particle alignment and mesocrystallization

Second, the distance between the particles was lowered by application of a strong external sedimentation force using a centrifugal field. Here, long extended fibers formed in a first aggregation step, which were nicely compacted toward partly highly organized threedimensional fiber structures. As judged by the very good packing structure and density on the mesoscale, this is evaluated to be the kinetically most controlled and most gentle approach toward mesocrystal formation.

In the third mode, a surface-active polymer was added that apparently adsorbs and stabilizes the $\{020\}$ faces of the nanoparticles, which form the tactosol. Besides defining shape and primary particle alignment, this also lowers the repulsive forces of the constituting components throughout superstructure formation. In spite of the simplicity of the approach, the structures are nevertheless very compact and highly defined. In our opinion, the data clearly illustrate the superiority of

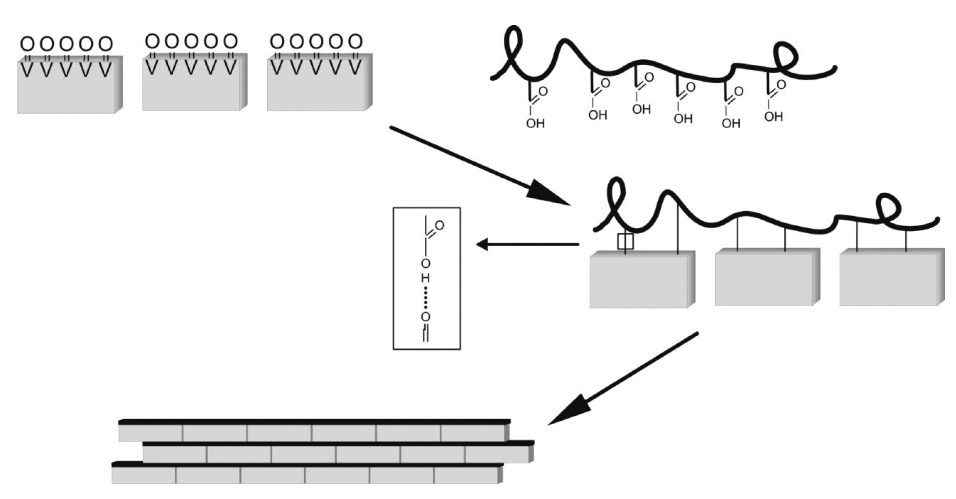

Scheme 1. Schematic view of $\mathrm{V}_{2} \mathrm{O}_{5}$ mesocrystal formation. It is assumed that the polymer adsorbs at the $\{020\}$ face of $\mathrm{V}_{2} \mathrm{O}_{5}$ particles and can align the secondary plates by hydrogen bridges. The repulsion of the original $\mathrm{V}_{2} \mathrm{O}_{5}$ layers is decreased by the polymer, and a pile of thin plates is formed (see text). For clarity reasons, the sulfonate groups of the polymer are not shown. the polymer approach, as compared to simple salt destabilization. Complementing the densification by centrifugation (which is in addition not a scalable technique and will be restricted to small sample specimens), we gain tensorial properties in the mesocrystal through the face specificity of adsorption, that is, an extra level of directionality.

Nanostructured materials have received great attention due to their unusual properties and potential applications. In particular, such materials are interesting for industrial applications. For example, vanadium pentoxide is 
used as a catalyst. The mesocrystalline material shown here might be useful for this application since a meso- crystal combines high crystallinity with high porosity and therefore larger specific surface area.

\section{MATERIALS AND METHODS}

Materials. Ammonium metavanadate $\left(\mathrm{NH}_{4} \mathrm{VO}_{3}\right.$, RiedeldeHaën), 1 M HCL (Merck), sodium chloride ( $\mathrm{NaCl}$, RiedeldeHaën), and the polymer ACUSOL 588G (acrylic acid/sulfonic acid copolymer, likely with 2-acrylamido-2methylpropanesulfonic acid, AMPS content from elemental analysis $=14.7 \mathrm{~mol} \%, M_{\mathrm{w}}=12000 \mathrm{~g} / \mathrm{mol}$ ), a well-known antiscale additive donated by Benckiser, were used without further purification.

Synthesis. The vanadium pentoxide tactosol was prepared according to Lagaly et al. ${ }^{40}$

In a bowl of agate stone, $1 \mathrm{~g}$ of $\mathrm{NH}_{4} \mathrm{VO}_{3}$ was grinded with few drops of distilled water and mixed with $10 \mathrm{~mL}$ of $1 \mathrm{M} \mathrm{HCl}$. The resulting red precipitate was transferred into a graduated cylinder. Afterward, it was filled up with distilled water to a total volume of $20 \mathrm{~mL}$. After sedimentation of the precipitate, the yellow supernatant solution was removed. Again, the precipitate was dispersed in distilled hot water $\left(80-90^{\circ} \mathrm{C}\right)$. The total volume was $20 \mathrm{~mL}$. Afterward, the precipitate was isolated. This process was repeated three times. Accordingly, the precipitate was removed once more and dispersed in hot water (total volume 20 $\mathrm{mL}$ ). The red dispersion was refilled with distilled hot water to a total volume of $40 \mathrm{~mL}$. After cooling to room temperature, a dilution series was prepared.

After about 3 or 5 days, a phase separation was observable, where the lower phase was the liquid crystal. Figure 8 shows a polarization microscope image of a $\mathrm{V}_{2} \mathrm{O}_{5}$ tactosol. The spindleshaped droplets are the tactoids. They have a size between 7 and $80 \mu \mathrm{m}$ depending on the age of the tactosol. One tactoid is obviously composed of millions of primary nanoparticles with 13 $\mathrm{nm}$ hydrodynamic diameter. The nanoparticles are arranged first to nanorods, which on the second level of structural hierarchy arrange in a typical nematic order to form the spindle-like tactoids with an average size of about $20 \mu \mathrm{m}$ (Figure 8). ${ }^{34}$

Three different experiments were carried out with the tactosol phases of different concentrations: (a) During dropwise addition of $13 \mathrm{~mL}$ of a $0.1 \mathrm{M}$ sodium chloride solution to the liquid crystal, the mixture was shaken until precipitation was observed This occurred at a final $\mathrm{NaCl}$ concentration of $56.5 \mathrm{mM}$. The resulting precipitate was freeze-dried. (b) The supernatant isotropic solution was carefully removed with the aid of a pipet. Afterward, the liquid crystal phase was treated by ultracentrifugation for $1 \mathrm{~h}$ at $55000 \mathrm{rpm}$ (SW 55 rotor). After decanting the supernatant solution from the sediment, the sediment was dried overnight in a desiccator under vacuum. (c) Then, $13 \mathrm{~mL}$ of ACUSOL $588 \mathrm{G}$ solution $(c=1 \mathrm{~g} / \mathrm{L}$ ) was added dropwise to the vanadium pentoxide tactosol. After addition of some drops of polymer solution, the mixture was shaken from time to time.

Characterization. The mesocrystals were characterized with different microscopy techniques. The light microscope images were obtained with a Leica microscope type DMR B (serial number: 172075 ) with and without crossed polarizers. The microstructure was observed by a scanning electron microscope (SEM) LEO 1550 Gemini system. The transmission electron microscope (TEM) images were recorded on a Zeiss EM $912 \Omega$ microscope operated at $120 \mathrm{kV}$. For the microtome cuts, the samples were cut into small pieces and embedded into LR white resin (medium grade). After a drying time of $5-7 \mathrm{~h}$, the samples were cut into microtome slices with a Leica Ultracut UCT. Analytical ultracentrifugation was carried out with a Beckman-Coulter XL-I analytical ultracentrifuge, thermogravimetry on a Netzsch TG 209 F1, and $\xi$-potential measurements on a Malvern Zetasizer.

Acknowledgment. We thank the Max Planck Society for financial support. The authors also thank R. Pitschke and H. Runge for the SEM and TEM measurements, as well as A. Völkel for the ultracentrifugation experiments. Dr. P. Bowen, EPFL Lausanne, and Dr. Uli Aschauer, Princeton University, are acknowledged for making the program Hamaker available to us for the DLVO cal- culations. Many thanks go to K. Beneke for discussion about the preparation of the tactosol and A. Schachtschneider for preparative assistance.

\section{REFERENCES AND NOTES}

1. Song, R. Q.; Cölfen, H. Mesocrystals-Ordered Nanoparticle Superstructures. Adv. Mater. 2009, 21, 1-30.

2. Cölfen, H.; Antonietti, M. Mesocrystals and Non Classical Crystallization; Wiley: New York, 2008.

3. Cölfen, H.; Antonietti, M. Mesocrystals: Inorganic Superstructures Made by Highly Parallel Crystallization and Controlled Alignment. Angew. Chem., Int. Ed. 2005, 44, 5576-5591.

4. Zhou, L.; O'Brien, P. Mesocrystals: A New Class of Solid Materials. Small 2008, 4, 1566-1574.

5. Oaki, Y.; Imai, H. Nanoengineering in Echinoderms: The Emergence of Morphology from Nanobricks. Small 2006, 2, 66-70.

6. Zhou, L.; Boyle, D. S.; O'Brien, P. Uniform $\mathrm{NH}_{4} \mathrm{TiOF}_{3}$ Mesocrystals Prepared by an Ambient Temperature SelfAssembly Process and Their Topotaxial Conversion to Anatase. Chem. Commun. 2007, 144-146.

7. Zhou, L.; Smyth-Boyle, D.; O'Brien, P. A Facile Synthesis of Uniform $\mathrm{NH}_{4} \mathrm{TiOF}_{3}$ Mesocrystals and Their Conversion to $\mathrm{TiO}_{2}$ Mesocrystals. J. Am. Chem. Soc. 2008, 130, 1309-1320.

8. Young, J. R.; Davis, S. A.; Bown, P. R.; Mann, S. Coccolith Ultrastructure and Biomineralisation. J. Struct. Biol. 1999, 126, 195-215.

9. Mann, S. Molecular Tectonics in Biomineralization and Biomimetic Materials Chemistry. Nature 1993, 365, 499-505.

10. Mann, S.; Davis, S. A.; Hall, S. R.; Li, M.; Rhodes, K. H.; Shenton, W.; Vaucher, S.; Zhang, B. J. Crystal Tectonics: Chemical Construction and Self-Organization Beyond the Unit Cell. J. Chem. Soc., Dalton Trans. 2000, 21, 3753-3763.

11. Mann, S.; Ozin, G. A. Synthesis of Inorganic Materials with Complex Form. Nature 1996, 382, 313-318.

12. Cusack, M.; Freer, A. Biomineralization: Elemental and Organic Influence in Carbonate Systems. Chem. Rev. 2008, $108,4433-4454$.

13. Hildebrand, M. Diatoms, Biomineralization Processes, and Genomics. Chem. Rev. 2008, 108, 4855-4874.

14. Omelon, S. J.; Grynpas, M. D. Relationships between Polyphosphate Chemistry, Biochemistry and Apatite Biomineralization. Chem. Rev. 2008, 108, 4694-4715.

15. Gower, L. B. Biomimetic Model Systems for Investigating the Amorphous Precursor Pathway and Its Role in Biomineralization. Chem. Rev. 2008, 108, 4551-4627.

16. Aizenberg, J.; Black, A. J.; Whitesides, G. M. Control of Crystal Nucleation by Patterned Self-Assembled Monolayers. Nature 1999, 398, 495-498.

17. Belcher, A. M.; Wu, X. H.; Christensen, R. J.; Hansma, P. K.; Stucky, G. D.; Morse, D. E. Control of Crystal Phase Switching and Orientation by Soluble Mollusc-Shell Proteins. Nature 1996, 381, 56-58.

18. Estroff, L. A.; Hamilton, A. D. At the Interface of Organic and Inorganic Chemistry: Bioinspired Synthesis of Composite Materials. Chem. Mater. 2001, 13, 3227-3235.

19. Kniep, R.; Busch, S. Biomimetic Growth and Self-Assembly of Fluorapatite Aggregates by Diffusion into Denatured Collagen Matrices. Angew. Chem., Int. Ed. Engl. 1996, 35 , 2624-2626.

20. Mukkamala, S. B.; Powell, A. K. Biomimetic Assembly of Calcite Microtrumpets: Crystal Tectonics in Action. Chem. Commun. 2004, 8, 918-919.

21. Tian, Z. R. R.; Voigt, J. A.; Liu, J.; McKenzie, B.; McDermott, 
M. J.; Rodriguez, M. A.; Konishi, H.; Xu, H. F. Complex and Oriented Zno Nanostructures. Nat. Mater. 2003, 2, 821-826.

22. Calderone, V. R.; Testino, A.; Buscaglia, M. T.; Bassoli, M.; Bottino, C.; Viviani, M.; Buscaglia, V.; Nanni, P. Size and Shape Control of $\mathrm{SrTiPO}_{3}$ Particles Grown by Epitaxial SelfAssembly. Chem. Mater. 2006, 18, 1627-1633.

23. Peng, Y.; Xu, A. W.; Deng, B.; Antonietti, M.; Cölfen, H. Polymer-Controlled Crystallization of Zinc Oxide Hexagonal Nanorings and Disks. J. Phys. Chem. B 2006 110, 2988-2993.

24. Qi, L. M.; Cölfen, H.; Antonietti, M.; Li, M.; Hopwood, J. D.; Ashley, A. J.; Mann, S. Formation of $\mathrm{BaSO}_{4}$ Fibres with Morphological Complexity in Aqueous Polymer Solutions. Chem.-Eur. J. 2001, 7, 3526-3532.

25. Legrouri, A.; Baird, T.; Fryer, J. R. Electron-Optical Studies of Fresh and Reduced Vanadium Pentoxide-Supported Rhodium Catalysts. J. Catal. 1993, 140, 173-183.

26. Nikolov, V.; Klissurski, D.; Anastasov, A. Phthalic-Anhydride from ortho-Xylene Catalysis - Science and Engineering. Catal. Rev.-Sci. Eng. 1991, 33, 319-374.

27. Ramirez, R.; Casal, B.; Utrera, L.; Ruizhitzky, E. Oxygen Reactivity in Vanadium Pentoxide-Electronic-Structure and Infrared-Spectroscopy Studies. J. Phys. Chem. 1990, 94, 8960-8965.

28. Wainwright, M. S.; Foster, N. R. Catalysts, Kinetics, and Reactor Design in Phthalic-Anhydride Synthesis. Catal. Rev.-Sci. Eng. 1979, 19, 211-292.

29. Colpaert, M. N. Exploratory Study of Catalytic-Oxidation Reactions on Vanadium Pentoxide Single-Crystals. Z. Phys. Chem. 1973, 84, 150-156.

30. El'nikova, L. V. Elastic Properties of Vanadium Pentoxide Aggregates and Topological Defects. J. Supercond. Novel Magn. 2008, 21, 473-478.

31. Kaznacheev, A. V.; Bogdanov, M. M.; Taraskin, S. A. The Nature of Prolate Shape of Tactoids in Lyotropic Inorganic Liquid Crystals. J. Exp. Theor. Phys. 2002, 95, 57-63.

32. Zocher, H. Über Freiwillige Strukturbildung in Solen. $Z$. Anorg. Allg. Chem. 1925, 147, 91-110.

33. Zocher, H. Taktosole Und Mesophasen. Kolloid Z. Z. Polym. 1954, 139, 81-85.

34. Sonin, A. S. Inorganic Lyotropic Liquid Crystals. J. Mater. Chem. 1998, 8, 2557-2574.

35. Livage, J. Vanadium Pentoxide Gels. Chem. Mater. 1991, 3 , 578-593.

36. Napper, D. Polymeric Stabilization of Colloidal Dispersions: Academic Press Inc.: London, 1983.

37. Kittaka, S.; Uchida, N.; Katayma, M.; Doi, A.; Fukuhura, M. Effect of Intercalation of Matal-lons on the Colloidal and Solid Properties of Vanadium Pentoxide Hydrate $\mathrm{V}_{2} \mathrm{O}_{5} \mathrm{NH}_{2} \mathrm{O}$. Colloid Polym. Sci. 1991, 269, 835-842.

38. Watson, J. H. L.; Heller, W.; Wojtowicz, W. Comparative Electron and Light Microscopic Investigations of Tactoid Structures in $\mathrm{V}_{2} \mathrm{O}_{5}$-Sols. Science 1949, 109, 274-278.

39. Freundlich, H.; Stapelfeldt, F.; Zocher, H. Quantitative Testings on Vanadin Pentoxydsol. I. The Anisotropy of Flow. Z. Phys. Chem. 1924, 114, 161-189.

40. Lagaly, G.; Schulz, O.; Zimehl, R. Dispersionen Und Emulsionen; Steinkopff: Darmstadt, Germany, 1997. 